Cahiers de recherches médiévales

\title{
Merlin et la scolastique
}

De la coincidentia oppositorum à la quaestio disputata dans Le Roman de Silence

\section{Silvère Menegaldo}

\section{(2) OpenEdition \\ 12 Journals}

Édition électronique

URL : https://journals.openedition.org/crm/2312

DOI : $10.4000 / \mathrm{crm} .2312$

ISSN : 1955-2424

Éditeur

Honoré Champion

\section{Édition imprimée}

Date de publication : 30 décembre 2005

Pagination : 211-225

ISSN : $1272-9752$

\section{Référence électronique}

Silvère Menegaldo, « Merlin et la scolastique », Cahiers de recherches médiévales [En ligne], 12 | 2005,

mis en ligne le 30 décembre 2008, consulté le 18 décembre 2022. URL : http://

journals.openedition.org/crm/2312 ; DOl : https://doi.org/10.4000/crm.2312

Ce document a été généré automatiquement le 18 décembre 2022.

Tous droits réservés 


\title{
Merlin et la scolastique
}

De la coincidentia oppositorum à la quaestio disputata dans Le Roman de Silence $^{*}$

\author{
Silvère Menegaldo
}

1 Dans le paysage romanesque de la seconde moitié du XIII ${ }^{e}$ siècle, Le Roman de Silence d'Heldris de Cornuälle ${ }^{1}$ occupe à n'en pas douter une place à part, et ce à plus d'un titre.

2 En premier lieu, l'œuvre d'Heldris, dont la forme versifiée à une telle époque peut déjà surprendre, n'appartient à aucune matiere bien définie, ni « de Rome », ni « de France », ni même « de Bretaigne », selon la tripartition bien connue de Jean Bodel. Il est vrai que Le Roman de Silence se déroule pour l'essentiel en Angleterre, fait intervenir le personnage de Merlin, et mentionne à trois reprises le nom d'Arthur (v. 109, 6154 et 6156) - sans qu'il soit possible d'ailleurs de situer à partir de là le moment du récit, sinon qu'il est postérieur à la naissance du roi. Mais cela ne suffit en aucun cas à en faire un roman « arthurien », et c'est à juste titre selon nous que S. Roche-Mahdi a fait disparaître de son édition cette épithète abusivement accolée par L. Thorpe à la sienne ${ }^{2}$.

Il s'agit ensuite, ce qui n'est pas si fréquent au Moyen Âge, d'un texte qui se donne pour une fiction ${ }^{3}$, mais où la multiplication des discussions, des débats entre personnages ou personnifications, sans compter les nombreuses interventions du narrateur, met l'accent sur un contenu idéologique particulièrement prégnant, sur la volonté d'engager sur certaines questions un débat d'idées, si bien qu'on pourrait parler, non pas de "roman à thèse ", mais de " roman idéologique ", dans le sens d'un roman qui se signale spécialement au lecteur comme porteur d'une réflexion, d'un questionnement d'ordre théorique ou philosophique ${ }^{4}$. Plus précisément, il s'agit dans Le Roman de Silence, pour simplifier, et sans considérer quelques débats annexes, d'une réflexion sur le statut de la femme dans la société médiévale, sur le caractère naturel ou culturel de l'identité sexuelle, et plus largement sur les rapports entre Nature et Culture ("Norreture », dans le texte), l'inné et l'acquis - l'attention que porte l'auteur à ce dernier point étant mise en évidence à l'occasion des divers débats entre ces deux abstractions personnifiées qui émaillent le texte.

4 Mais en même temps - et c'est là sa dernière particularité - Le Roman de Silence n'est justement pas un "roman à thèse ", mais un roman dont le sens ou les intentions 
semblent échapper, qui ne cherche apparemment pas à trancher les débats qu'il met en scène, particularité que rendent sensible les interprétations très divergentes dont l'œuvre d'Heldris a été l'objet, œuvre dont la critique a pu tour à tour souligner la misogynie, le féminisme ou plus souvent encore la remarquable ambiguïté - P. L. Allen qualifiant ce texte, en une formule éclairante, de "fiction that is all about ambiguity " À cet égard il faut préciser que Le Roman de Silence jouit aussi d'une situation assez exceptionnelle eu égard au flot critique dont il a été la source depuis quelques années, en particulier outre-Atlantique, les questions qu'il pose en faisant pour les feminist ou gender studies un terrain d'élection ${ }^{6}$.

\section{Le problème de la fin}

Suite à un décret du roi d'Angleterre, Ebain, interdisant à toute femme d'hériter, Cador, le neveu du roi, et Eufemie, sa femme, décident dès sa naissance d'élever leur fille Silence comme un garçon. Sans nous attarder sur les différentes péripéties du roman, rappelons simplement que le parcours de Silence permet non seulement sa transformation progressive et finalement totale en homme, mais également son accomplissement sous la figure du plus vaillant des chevaliers. Cependant, à la cour du roi Ebain, Silence est poursuivie des assiduités de la reine Eufeme ; confrontée au refus obstiné de Silence, la reine veut s'en débarrasser en l'envoyant quérir Merlin, qui d'après une prophétie ne saurait être capturé que par une femme. Une fois amené à la cour, Merlin révèle toute la vérité devant le roi : Silence est une femme, la reine est adultère, en conséquence de quoi le roi la fait écarteler et épouse Silence.

Comment comprendre la fin du roman ? Il s'agit semble-t-il d'un retour à l'ordre sexuel et naturel, puisque Silence retrouve son sexe de femme et épouse le roi. Cependant, comme le rappelle Ebain lui-même au moment précis où la vérité se fait jour :

«Silence, moult as esté prols,

Bials chevaliers, vallans et buens ;

Mellor n'engendra rois ne cuens ». (v. 6579-6581)

7 Tout le parcours qui a précédé, et qui a trouvé son aboutissement dans la figure accomplie du chevalier " vallans et buens ", peut-il être réduit à rien, et Silence cesser d'être un homme pour redevenir aussitôt une femme ? Ce parcours peut-il être effacé, comme s'il n'avait jamais eu lieu, de même qu'il suffit à Nature d'effacer le "halle » (v. 6674) de la peau de Silence pour faire disparaitre « Tolt quanque ot sor le cors de malle» (v. 6673) - effacement de surface finalement, qui dit bien le retour à une apparence féminine de Silence, mais peut-être rien d'autre ? Est-ce bien la victoire de Nature (le sexe féminin) sur Culture (l'éducation masculine) ? Est-ce la manifestation d'une idéologie misogyne, qui refuserait finalement à la femme toute possibilité de sortir de sa condition, ou au contraire cette possibilité subsiste-t-elle, que l'ensemble du roman s'est plu à illustrer, en dépit du sort final de Silence?

8 À dire vrai, ce qui se donne pour la conclusion de l'auteur, maistre Heldris, n'aide guère à répondre à cette question :

Maistre Heldris dist chi endroit

C'on doit plus bone feme amer

Que haïr malvaise u blasmer.

Si mosterroie bien raison:

Car feme a menor oquoison

Por que ele ait le liu ne l'aise 
De l'estre bone que malvaise.

S'ele ouevre bien contre nature,

Bien mosterroie par droiture

C'on en doit faire gregnor plait

Que de celi qui le mal fait.

Se j'ai jehi blasmee Eufeme

Ne s'en doit irier bone feme.

Se j'ai Eufeme moult blasmee

Jo ai Silence plus loëe.

Ne s'en doit irier bone fame,

Ne sor li prendre altrui blasme,

Mais efforcier plus de bien faire. (v. 6684-6701)

Conclusion on ne peut plus ambiguë, misogyne et du côté de Nature, puisque la femme est donnée comme mauvaise par nature, et n'en peut mais ; en même temps féministe et du côté de Culture, puisque la femme peut aussi être vertueuse, en allant précisément contre sa mauvaise nature ; mais peut-être, en dernier ressort, avant tout pragmatique, dans la mesure où Heldris semble avant tout manifester le souci de ne pas froisser son public, et particulièrement les bones femes qui pourraient en faire partie ${ }^{7}$.

La fin ne semble donc pas apporter de solution aux enjeux idéologiques du roman, et c'est une particularité qui apparaît d'autant plus remarquable lorsque que l'on compare Le Roman de Silence à d'autres textes faisant intervenir un scénario similaire de travestissement d'une femme en homme ${ }^{8}$, mais dont l'aboutissement est bien différent. D'un côté, dans Aucassin et Nicolette par exemple, on a affaire au déguisement temporaire de Nicolette en jongleur (donc en homme), travestissement qui occupe un moment beaucoup trop court dans la vie du personnage pour aboutir à une quelconque transformation ; ainsi il apparaît également que les problèmes posés par la fin du Roman de Silence sont étroitement liés à l'organisation de son intrigue, où Silence est non seulement travestie, mais aussi éduquée comme un homme, dès sa naissance et pendant des années. À l'inverse, dans les quelques récits que l'on connaît où le travestissement est durable, dans Tristan de Nanteuil, et dans Yde et Olive surtout ${ }^{9}$, l'héroïne finit effectivement transformée en homme, suite à une intervention divine, ce qui règle finalement toute espèce de problème concernant la cohabitation de traits masculins et féminins dans un même personnage qui, devenant définitivement un homme, en même temps cesse définitivement d'être une femme. Entre travestissement temporaire et transformation définitive, Le Roman de Silence occupe donc une place originale, pour la raison enfin que le devenir de Silence est au cœur de l'œuvre, constitue son véritable sujet; ce qui n'est pas le cas par exemple de l'histoire de Grisandole, court récit appartenant au pan narratif précédant le Lancelot en prose (Suite $d u$ Merlin ou Premiers faits $d u$ roi Arthur $)^{10}$, et dont le déroulement manifeste une proximité particulièrement étroite avec le roman d'Heldris : en effet, insérée dans un vaste ensemble où dominent les figures d'Arthur et de Merlin, il est évident que l'histoire de Grisandole ne s'intéresse pas tant à Grisandole elle-même qu'au prophète arthurien qu'elle met en scène ${ }^{11}$.

\section{En quête du sens : voix et personnages autorisés}

11 Considérant que ni la fin du roman, ni la conclusion de maistre Heldris n'apportent de résolution claire aux questions qu'il pose, il apparaît alors nécessaire de reporter le regard sur l'ensemble du texte, et d'essayer de déterminer les éléments sur lesquels 
s'appuyer afin de parvenir à une compréhension satisfaisante des intentions de l'auteur, à un sens si possible explicitement donné, et qui parait d'autant plus attendu que le roman ne cache pas ses intentions réflexives, son caractère nettement « idéologique ».

Sans revenir en détail sur une question que nous avons eu l'occasion d'aborder dans notre thèse ${ }^{12}$, nous voudrions seulement rappeler en guise de préliminaires quelques points concernant l'inscription de l'idéologie dans le texte littéraire :

(1) on peut prêter à tout roman (aussi bien, à tout texte) une portée idéologique et l'intention de susciter chez son lecteur une certaine conviction, aussi bien envers l'univers fictionnel qu'il construit qu'envers le système de valeurs mis en place dans cet univers ${ }^{13}$.

(2) cette idéologie textuelle peut relever de différents degrés de "partageabilité » - si l'on nous passe cet horrible néologisme -, selon la portée des valeurs considérées, et la capacité du lecteur à les partager ou non. Ainsi dans Le Roman de Silence il est possible de distinguer entre valeurs universelles, ou que l'on peut présumer telles, comme la condamnation du meurtre prémédité que manigancent les deux jongleurs à l'encontre de Silence; valeurs historiques et culturelles, en l'occurrence propres à la culture médiévale occidentale, comme les valeurs chrétiennes, ou la misogynie par exemple, nettement affirmée dans le roman, comme dans beaucoup d'autres textes médiévaux d'ailleurs, et qu'il ne s'agit pas de ce fait d'appréhender comme on pourrait le faire dans un roman d'aujourd'hui ; valeurs historiques et culturelles, et spécifiques en outre à certains groupes sociaux, comme l'exigence de vaillance, de générosité etc. caractéristique de l'idéologie aristocratique médiévale; valeurs particulières, individuelles enfin, propres à tel auteur ou telle œuvre, comme c'est probablement le cas - nous y reviendrons plus loin - du pragmatisme dans Le Roman de Silence.

(3) l'idéologie peut également manifester différents degrés de cohésion ou de dispersion, d'univocité ou d'équivocité, de la plus concentrée («message » simple, univoque, réitéré à de nombreuses reprises) à la plus diluée (significations contradictoires, dispersées etc.), jusqu'à une véritable " polyphonie » idéologique ${ }^{14}$.

(4) l'idéologie est véhiculée par différentes voix ou supports, plus ou moins autorisés (c'est-à-dire que l'on peut considérer comme les porteurs plus ou moins avérés, fiables, du sens), depuis l'auteur parlant en son nom propre, le narrateur représentant ou non de l'auteur, les différents personnages, leurs discours ou leurs actes, l'intrigue en dernier ressort, dont le déroulement même importe évidemment à la construction du sens.

(5) enfin le travail textuel de l'idéologie suppose un va-et-vient constant entre l'auteur, le texte et le lecteur, confrontant ses propres valeurs avec celles du texte, à partir desquelles il peut inférer celles de l'auteur.

13 À partir de ce cadre rapidement posé, si l'on tente de dresser un tableau idéologique du Roman de Silence, en se limitant dans le cas présent aux diverses voix, c'est-à-dire aux divers avis qui trouvent à s'exprimer sur la question centrale du roman (qui se signale en tant que telle par l'abondance des discours consacrés au statut ou à l'image de la femme, aux relations entre Nature et Culture), on ne peut que constater, à côté de redondances caractéristiques selon S. R. Suleiman du "roman à thèse ", un jeu de contradictions presque systématique.

Il faut évidemment commencer par le narrateur, voix essentielle à la construction du sens dans l'exercice de sa «fonction idéologique», celle qui lui permet, selon 
G. Genette, la formulation d'un « commentaire autorisé de l'action » ${ }^{15}$, ou encore, pour citer V. Jouve, de « jugements explicites qui prennent parfois la tournure de maximes intemporelles $»^{16}$. Sans distinguer ici, comme nous avons pu le faire dans notre thèse, entre narrateur et auteur inscrit (c'est-à-dire le maistre Heldris des vers 1 et 6684), ni entre narrateur explicite (que signale notamment le recours à la première personne du singulier et/ou au présent gnomique) et implicite, il suffira de souligner le caractère nettement contradictoire de l'ensemble des propos pouvant être référés, de la façon la plus large, au narrateur. À plusieurs reprises en effet ce dernier semble trancher en faveur de Nature (par exemple v. 2295-2298 ou 2423-2424), et ne se prive pas d'un certain nombre de réflexions nettement misogynes, en particulier à propos d'Eufeme (v. 3901-3924, 4157-4158, 4266-4269, etc.). Cependant, c'est le même narrateur que l'on voit défendre les pouvoirs de Culture et de l'éducation, bonne (v. 5165-5166) ou mauvaise (v. 2339-2342), et délivrer des éloges à Silence devenue chevalier « vallans et buens » (v. 5179).

Dans un second temps, on pourra considérer à part les deux débats entre personnifications de Nature et de Culture (auxquels on peut ajouter le monologue de Nature des v. 2266-2294), lieux plus particulièrement attendus d'un positionnement idéologique, dans la mesure où ils constituent la manifestation explicite des ambitions réflexives du roman, se situant sur un plan qui dépasse en quelque sorte celui de l'intrigue proprement dite, même si leur rôle est aussi de figurer l'intériorité tourmentée de Silence ou de Merlin. Pourtant, là encore, le débat reste purement contradictoire, en suspens, sans solution. Le premier débat (v. 2500-2604), qui a lieu au moment où Silence a atteint l'âge de douze ans, sans voir la victoire de Nature ou de Culture, reste suspendu, le dernier mot revenant finalement à Raison, qui amène Silence à reconnaître de façon toute pragmatique que «miols valt li us d'ome/Que l'us de feme, c'est la some» (v. 2637-2638). Quant au second débat (v. 5994-6089), qui concerne cette fois Merlin, loin de s'interroger sur l'opposition logique entre l'inné et l'acquis, il dérive en fait sur le plan axiologique, puisqu'il porte sur l'origine du mal, que les deux abstractions personnifiées s'attribuent réciproquement, ce qui revient à opposer une Nature ou une Culture fondamentalement bonnes à une Nature ou une Culture fondamentalement mauvaises. On observe d'ailleurs le même phénomène de glissement dans les réflexions du narrateur faisant suite au monologue de Nature (v. 2295-2342).

16 Enfin les personnages eux-mêmes, plus ou moins autorisés selon les cas, tiennent également les propos les plus contradictoires. Ainsi le roi Ebain, figure pourtant autorisée par son statut et par l'éloge qu'en fait le narrateur, notamment quand il le qualifie de «bon home et sage/Et atenpret de son corage » (v. 4187-4188; voir aussi les v. 107-130 ou 1302-1308), tient les propos les plus flatteurs à l'égard d'Eufeme, au début du roman (v. 182-184), ou de Silence, à la fin (v. 6630-6636), dans des termes qui reprennent d'ailleurs ceux du narrateur (les vers 6579-6581, calqués sur les vers 5179-5180) ; cependant, outre que l'éloge même puisse apparaître ambigu (s'il « n'est si preciose gemme/Ne tels tresors com bone feme », v. 6633-6634, c'est donc qu'elles sont fort rares), le roi ne craint pas par ailleurs les réflexions les plus misogynes, notamment dans la tirade adressée à la reine qui s'ouvre sur "Sens de feme gist en taisir » (v. 6398). Il en est de même pour «Cador le preu, ki moult savoit » (v. 392), le père de Silence, que son amour pour Eufeme n'empêche pas de critiquer les femmes (v. 667-675), tandis qu'une fois marié, il s'appuie sur la Genèse pour souligner : 
" Entr'ome et feme a grant commune,

Car d'als .ii. est la sustance une ». (v. 1711-1712) elle toutes les contradictions du roman. Élevée comme un garçon, elle connaît à l'âge de douze ans son unique crise, qui donne lieu au premier débat entre Nature et Culture, à l'issue duquel Silence, agitée de "diverse cogitatiön» (v. 2663), demeure «le cuer diviers» (v. 2681); puis elle endosse le costume de jongleur, métier apparemment choisi parce qu'il représente une sorte de moyen terme entre activités féminines et masculines (v. 2863-2869); enfin elle devient chevalier, tout en restant jusqu'à la fin à la fois homme et femme, «vallés mescine » (v. 3763), comme la désigne Heldris. Et c'est en vertu de cette coexistence des sexes que Silence peut apparaitre conjointement comme l'« ouvre forcible » (v. 1807), le chef-d'œuvre de Nature, et la manifestation du pouvoir de Culture, non seulement lorsqu'elle sera devenue chevalier, mais aussi dès son enfance, avec ce curieux paradoxe d'un « enfes » naturellement disposé à l'éducation, et qui « se doctrine » tout seul :

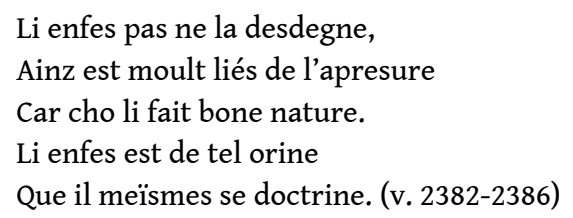

18 En ce point du texte, le débat entre Nature et Culture apparaît même entièrement neutralisé, rendu caduc par la présence en Silence d'une qualité qu'aucun poète médiéval ne s'aviserait de vraiment remettre en cause, son "orine ", ou sa "bonté » (v. 2395), c'est-à-dire sa noblesse (voir aussi les v. 3195-3204 opposant la « francise » de Silence à la « culvertise » des jongleurs).

On pourrait multiplier les exemples d'avis contradictoires exprimés dans le roman, parfois par des personnages d'importance très secondaire, tel le chancelier du roi Ebain (v. 5001-5016). Ceux que nous avons relevés, cependant, devraient suffire à manifester comment Le Roman de Silence, en accumulant sur la question de la femme ou de la primauté relative de Nature et de Culture les points de vue les plus opposés, parvient à un véritable brouillage du sens, que reflète d'ailleurs l'intrigue elle-même, non seulement dans sa conclusion, mais aussi dans son déroulement, notamment par la mise en scène de personnages antithétiques comme Eufemie et Eufeme.

\section{La solution Merlin}

Reste un personnage dont nous n'avons guère parlé jusqu'à maintenant, Merlin ${ }^{17}$, qui fait son apparition à la fin du roman pour révéler au roi le sexe réel de Silence et l'adultère de la reine. En dehors même de la conclusion qu'il permet d'apporter à l'intrigue, on va voir que le travail opéré sur ce personnage et les traits qui le constituent par l'auteur du Roman de Silence tend à signaler Merlin comme la véritable clé interprétative de ce texte. Quoiqu'à première vue il apparaisse comme un personnage parfaitement exogène, deus ex machina intervenant de façon parfaitement imprévisible ${ }^{18}$, emprunté à une matière arthurienne qui n'est pas celle du roman, si l'on compare néanmoins le Merlin d'Heldris à celui des romans en prose, et particulièrement à celui de l'histoire de Grisandole, source supposée de notre roman, on s'aperçoit d'abord que Merlin est doté de traits particuliers qui le distinguent de ses 
incarnations en prose, ensuite qu'il est en fait parfaitement intégré à l'intrigue du Roman de Silence.

Certes entre la fin du Roman de Silence et l'histoire de Grisandole relatée dans la Suite du Merlin la parenté est manifeste et a déjà été nettement mise en évidence ${ }^{19}$, et il est parfaitement possible, sinon certain, que le roman en vers se soit inspiré du roman en prose $^{20}$, mais il n'en reste pas moins que certains éléments propres au récit d'Heldris trahissent également des emprunts à d'autres textes de la tradition merlinienne, plus anciens, et en particulier à la Vita Merlini de Geoffroy de Monmouth ${ }^{21}$. Comme dans la Vita en effet, Merlin apparaît dans Le Roman de Silence comme un personnage autonome, qui n'est pas spécifiquement lié au règne d'Arthur, quand bien même son rôle dans la conception du roi serait, rapidement, rappelé. Ainsi affranchi de tout rôle historique, mis à l'écart de la société et du monde, le personnage peut retrouver d'autant plus facilement deux de ses traits fondamentaux, son état d'homme sauvage, et la folie qui l'habite. De fait la Vita est d'abord l'histoire d'une folie, puisque dès le premier vers Geoffroy manifeste son intention de chanter «rabiem musamque jocosam », la folie et l'histoire plaisante de Merlin. Et c'est un premier accès de folie, après la mort de ses frères, qui fait de lui un "silvester homo" (v. 80). Ensuite, en dépit de quelques moments de rémission, Merlin est encore régulièrement frappé de crises de démence, qui jouent un rôle essentiel dans toute la suite du texte, jusqu'à sa guérison finale. C'est le trait qui caractérise le plus nettement le personnage, et qui apparaît indissociablement lié à son état d'homme sauvage et sa capacité à révéler la vérité (à la fin de la Vita d'ailleurs, Merlin guéri de sa folie semble aussi avoir perdu ses pouvoirs prophétiques). De même dans Le Roman de Silence Merlin apparaît d'emblée sous les traits de l'homme «salvages » (v. 5799) ${ }^{22}$, état allant de pair avec sa folie, même si cette dernière ne lui est pas conférée comme une qualité objective par le narrateur, à la manière de la Vita, mais comme le constat d'un regard subjectif porté par les personnages du roman. Quand après l'avoir capturé Silence conduit Merlin en ville puis à la cour, tous, aussi bien la foule que les nobles et le roi, le considèrent, en dépit de ses talents reconnus de prophétie, comme un «sot» (v. 6185 et 6278) ou un «fol» (v. 6303). À la différence de la Vita, le fait qu'il s'agisse d'une évaluation subjective effectuée par les personnages du roman permet certainement de ménager plus aisément le retournement qui amènera à la révélation de la vérité. Il n'empêche qu'avant cette révélation, Merlin est non seulement considéré, mais même traité comme un fou, sans aucun ménagement, maltraité, molesté, puis jeté en prison et menacé de mort, de même que dans la Vita il est emprisonné à plusieurs reprises, à cause de son refus obstiné de rester à la cour du roi Rodarch. Rien de tel en revanche dans l'histoire de Grisandole, où le prophète a droit à beaucoup plus d'égards. S'il apparaît bien comme un homme sauvage, à aucun moment il n'est qualifié de fou ${ }^{23}$, emprisonné ni même véritablement maltraité. On peut légitimement supposer que l'importance conférée à Merlin dans les romans en prose du Graal était difficilement compatible avec certains des éléments contenus notamment dans la Vita. À l'inverse, en ayant recours à ces éléments, et en particulier à la folie, pour caractériser son personnage de Merlin, Heldris manifeste clairement son indépendance vis-à-vis du domaine arthurien. L'auteur du Roman de Silence n'agit donc pas en plagiaire, mais, en revenant aux thèmes fondamentaux de la Vita, s'est livré à un véritable travail de composition sur son personnage, ainsi doté d'une couleur propre, où se trouvent plus particulièrement mis en avant l'état d'homme sauvage et la folie qui lui est associée. 

est revenu à son état sauvage, si l'on peut dire, qui permet d'intégrer pleinement Merlin au Roman de Silence, en le faisant participer au débat entre Nature et Culture. En effet, Merlin homme sauvage, ou plus précisément homme autrefois civilisé devenu sauvage, puis devant retourner à la civilisation, se trouve pris comme Silence dans un débat entre Nature et Culture, la première représentant son ancien état d'homme civilisé, la seconde son nouvel état d'homme sauvage; et c'est la victoire de Nature, après que Merlin a cédé aux attraits de la viande cuite, du miel, du lait et du vin, qui permet sa capture et son retour temporaire à la civilisation.

Se distinguant donc d'éventuels modèles en prose, il apparaît finalement qu'Heldris retourne à l'ambivalence première et fondamentale de Merlin, à la fois homme et animal, civilisé et sauvage, sage et fou, unissant en lui les caractères les plus contradictoires, ce Merlin qui, comme le rappelle P. Walter, "offre dès son état d'enfant l'image la plus accomplie de la coexistence des contraires (coincidentia oppositorum) $\aleph^{24}$. Ainsi Merlin peut-il apparaître comme le véritable miroir de Silence, aussi ambivalent qu'elle peut l'être. De fait Heldris ne se prive pas de souligner plus ou moins explicitement les divers aspects que les deux personnages ont en commun : tous deux ont été - et ici l'utilisation d'une expression identique est assez notable - «norri en bois » (vers 2354 et 6003$)^{25}$, ce qui en fait des personnages à la fois marginalisés, mais exceptionnels, notamment par leur ambivalence (sagesse et folie, masculin et féminin), qui trouve à se traduire sur le même plan idéologique par un débat entre Nature et Culture; en outre Merlin et Silence ont vis-à-vis l'un de l'autre une fonction parfaitement analogue de révélateur révélé, car chacun au contact de l'autre est confronté à l'ambiguïté de son propre état, se révèle et révèle l'autre pris dans un débat entre Nature et Culture.

Par ailleurs Merlin est aussi un personnage particulièrement autorisé, peut-être même le personnage le plus autorisé du roman, statut particulier qui se ressent à plusieurs niveaux: intertextuel d'abord, avec le prestige rattaché à une importante tradition, rapidement évoquée par Heldris (tour de Vortigern, conception d'Arthur), qui tout en conservant vis-à-vis d'elle toute sa liberté, n'en reconnaît pas moins l'existence; intradiégétique ensuite, étant donné le rôle joué par Merlin dans la révélation de la vérité, mais aussi dans le déroulement de l'intrigue, dans la mesure où il apparaît évident que c'est Merlin lui-même, sous les traits d'un "hom tols blans al dos" (v. 5875), qui organise sa propre capture par Silence; d'où une dernière source d'autorité, au niveau extradiégétique cette fois, la similitude des rôles qui tend à faire de Merlin un double de l'auteur (ce qui pour le coup ne peut que rappeler le personnage des romans du Graal en prose).

Cependant, tout autorisé qu'il soit, la particularité de Merlin est paradoxalement de ne pas se prononcer. En effet, après avoir été capturé par Silence, Merlin rit à plusieurs reprises devant divers spectacles, en ville, puis devant le roi Ebain et la reine, mais refuse obstinément de donner la raison de ces éclats, et même de prononcer un seul mot :

Com plus l'enquierent, plus se taist.

Tant li delite li taisirs

Que parlers li est nonplaisirs. (v. 6280-6282)

Menacé de mort, Merlin finit par parler, mais se contente de révéler en quelque sorte machinalement la vérité, une vérité de l'ambivalence ou de l'opposition entre être et 
apparence, sans formuler aucune espèce d'opinion sur les débats qui agitent le roman sans "mesdire de feme» (v. 6372), en dépit des reproches injustifiés de la reine, ce qu'on attendrait pourtant, étant donné le contenu de ses révélations. Il se limite en fait à un bref éloge de Silence (v. 6541-6544), sans qu'on sache le moins du monde dans quel sens il va, celui de Nature ou celui de Culture.

Enfin, comme son opinion, le sort de Merlin lui-même reste en suspens à la fin du roman. Le texte le mentionne une dernière fois, quand le roi Ebain doute encore de ses révélations sur Silence :

Li rois en est encor en dolte.

Fait Merlin fermement tenir. (v. 6568-6569)

C'est le dernier vers concernant Merlin, qui laisse semble-t-il tout à fait volontairement le devenir du prophète en suspens. On pourrait certes comprendre que Merlin tenu «fermement» ne retournera pas dans les bois et restera acquis à sa Nature initiale d'homme civilisé ; ou inversement que Merlin, personnage fuyant, « fuitils » (v. 5800), comme le qualifie Eufeme, doit être "fermement» retenu pour ne pas chercher à retourner à sa Culture d'homme des bois, comme il le fera finalement dans la Vita Merlini. Mais en fait le texte n'opte pour aucune des deux solutions : pas plus pour le personnage de Merlin que pour celui de Silence le débat entre Nature et Culture ne trouve sa conclusion à la fin du roman.

\section{Suspension du sens et multiples niveaux de lecture}

Ainsi le personnage de Merlin, dans son discours (ou plutôt son absence de discours) et dans son être même, joue selon nous un rôle essentiel pour confirmer que le roman n'entend pas trancher le débat, qu'il multiplie les points de vue contradictoires sans chercher à résoudre les contradictions. Même si Le Roman de Silence ne cache pas sa portée idéologique, il relève en même temps d'une logique de suspension du sens, ou d'irrésolution, quel que soit d'ailleurs le niveau de lecture ou d'interprétation auquel on se situe ; car il est possible d'envisager cette suspension du sens à plusieurs niveaux, dont on peut considérer que là encore ils se trouvent en quelque sorte figurés dans le personnage de Merlin.

En effet Merlin, en tant qu'être surnaturel et foncièrement ambivalent, répond à un premier niveau d'interprétation, où l'on retrouve d'ailleurs la marque du substrat mythique ou folklorique dans lequel une bonne part de la littérature médiévale plonge ses plus profondes racines, c'est le niveau de la "pensée sauvage ", pour reprendre la formule de C. Lévi-Strauss, qui se manifeste ici à travers un de ses aspects caractéristiques: «loi de non-contradiction » ou "loi de participation» définie par L. Lévy-Bruhl ${ }^{26}$ notamment dans Les Fonctions mentales dans les sociétés inférieures et dans La Mentalité primitive, ou bien coincidentia oppositorum étudiée par M. Éliade dans "Méphistophélès et l'androgyne " ${ }^{27}$, l'une comme l'autre mettant le même principe en évidence, à savoir la possibilité de l'union non contradictoire des contraires. De fait la constitution des personnages de Merlin et de Silence, le caractère protéen du premier, l'androgynie du second apparaissent bien obéir à un tel principe, de même finalement que le roman tout entier, qui fait cohabiter, sans résolution, des points de vue parfaitement contradictoires sur la femme ou la primauté relative de Nature et de Culture ${ }^{28}$. 
Cependant Merlin ne fait pas seulement signe vers ce premier niveau de lecture. De même que le titre de maistre attaché à Heldris laisse entendre que l'auteur du Roman de Silence a dû faire un passage par l'université, de même Merlin, par le savoir dont il dispose, n'est pas sans relation avec le domaine universitaire ${ }^{29}$, et peut suggérer un second niveau de lecture, le niveau rationnel et intellectuel cette fois. Vu l'époque probable où a été rédigé le roman, époque où l'enseignement universitaire connaît son plein développement, il n'est pas interdit de retrouver dans l'organisation idéologique du Roman de Silence quelque chose de la scolastique, et notamment de la quaestio disputata qui, comme le rappelle B.C. Bazàn, devient à partir du XIII ${ }^{\mathrm{e}}$ siècle " un instrument de travail et de recherche d'usage généralisé dans l'Europe intellectuelle médiévale $»^{30}$. De fait le roman peut apparaître conçu sur le modèle d'une quaestio, portant sur la primauté relative de Nature et de Culture ${ }^{31}$ : une fois le problème posé, dès la naissance de Silence, l'auteur, parfois par le biais de personnages jouant, comme dans une véritable séance universitaire, le rôle d'opponens ou de respondens, accumule les arguments pour ou contre Nature et Culture. Il manque seulement, comme à la fin d'une quaestio disputata, la determinatio du maître, censée trancher le débat dans un sens ou l'autre ; il n'était pas rare cependant, dans le cadre de l'enseignement universitaire, que cette conclusion manquât, ou fût remise à une séance ultérieure; dans ce cas, la quaestio consiste seulement dans l'affrontement et la discussion de points de vue contradictoires, sans chercher à trancher, comme le fait Le Roman de Silence.

Mais il reste un dernier niveau d'interprétation, peut-être le plus révélateur des intentions de l'auteur, et qui n'est pas le moins étonnant, la lecture pragmatique, que peut figurer encore Merlin, dans la mesure où son intervention de deus ex machina relève elle-même d'une sorte de pragmatisme narratif. De fait, comme l'a bien relevé $\mathrm{H}$. Lloyd ${ }^{32}$, le pragmatisme ${ }^{33}$ est une attitude caractéristique de plusieurs personnages du roman, et de l'auteur lui-même, comme on l'a vu dans sa conclusion. L'exemple le plus frappant concerne certainement Silence elle-même, dont l'hésitation entre Nature et Culture est finalement vaincue par un argument on ne peut plus pragmatique de Raison:

[...] miols valt li us d'ome

Que l'us de feme, c'est la some. (v. 2637-2638)

Plus largement, qu'il s'agisse de rois, de jongleurs, ou d'un mari et de sa femme, on notera la propension de la plupart des personnages à discuter, à exprimer, à confronter différents points de vue pour adopter la meilleure attitude face à une situation donnée. Ainsi Le Roman de Silence apparaît dans une certaine mesure promouvoir une forme originale de pragmatisme, dont le jugement final de maistre Heldris sur les femmes pourrait bien relever.

\section{Conclusion}

"Roman idéologique », Le Roman de Silence semble multiplier à plaisir les points de vue contradictoires sur une question donnée, dans une logique qui n'est pas sans rappeler celle du Roman de la Rose de Jean de Meun, où de même les propos les plus misogynes s'accompagnent des excuses de l'auteur auprès des "vaillanz fames » (v. 15199), et où d'un côté "Trop est grant force que nature/Qu'el passe neïs norreture" (v. 14041-14042), mais de l'autre les hommes, «Par norreture nete et fine» (v. 17082) peuvent «Leurs meurs naturels refrene[r] » (v. 17090); mais faut-il s'en étonner, dans 
la mesure où "Ainsi va des contraires choses:/Les unes sont des autres gloses" (v. 21577-21578) ${ }^{34}$ ? Certainement moins «idéologique » que le texte de Jean de Meun, Le Roman de Silence, qui est d'abord un roman, n'en apparait pas moins, en plein XIII ${ }^{e}$ siècle, caractéristique d'une époque où peuvent encore se croiser et se recouvrir divers modes de pensée, divers modes d'appréhension du réel, plus ou moins anciens ou nouveaux, plus ou moins élaborés, sans que l'un soit exclusif des autres; de même l'auteur n'entend pas trancher dans le débat entre Nature et Culture, non plus que se cantonner au seul registre sérieux de la réflexion théorique, dans un roman où l'humour et le rire, il ne faudrait pas l'oublier, sont loin d'être absents ${ }^{35}$. Pour Heldris comme pour le lecteur, la superposition des niveaux de sens, et la conscience de cette superposition, est aussi une invite à une attitude pragmatique, libre de se construire sa propre opinion, voire de ne pas en avoir et de se laisser porter par les détours de la fiction - à moins qu'il ne faille finalement s'en remettre (en dépit du relatif désengagement que semble manifester Heldris sur ce point) à la vérité la plus incontestable, celle de la foi chrétienne.

\section{NOTES}

* Ce titre par sa formulation est façon de rendre un modeste hommage aux travaux de M. Stanesco, source constante de réflexion, et plus particulièrement à l'un d'entre eux, "Nigromance et Université. Scolastique du merveilleux dans le roman français du Moyen Âge » (1987, repris dans D'armes et d'amours. Études de littérature arthurienne, Orléans, Paradigme, 2002, p. 261-279), dans la perspective duquel cet article voudrait se situer.

1. Notre édition de référence sera celle de S. Roche-Mahdi (1992), actuellement la plus accessible. Pour la liste des autres éditions et traductions, ainsi que pour tout le détail des références concernant les études critiques, nous nous permettons de renvoyer à la bibliographie que nous avons établie avec D. James-Raoul.

2. Sur la question du mélange ou de l'incertitude des matières dans notre roman, voir R. Trachsler, Disjointures-Conjointures. Étude sur l'interférence des matières narratives dans la littérature française du Moyen Âge, 2000, p. 118-126.

3. Voir les propos quelque peu contradictoires d'Heldris, prétendant traduire une "estorie» latine «en romans", sans s'interdire d'ajouter «Avoic le voir sovent mençoigne/Por le conte miols acesmer » (v. 1660-1665).

4. Nous nous inspirons ici de la définition préparatoire proposée par S. R. Suleiman, dans Le Roman à thèse ou l'autorité fictive (Paris, PUF, 1983), du « roman à thèse », à savoir un roman « qui se signale au lecteur principalement comme porteur d'un enseignement, tendant à démontrer la vérité d'une doctrine politique, philosophique, scientifique ou religieuse » (p. 14).

5. P. L. Allen, "The Ambiguity of Silence. Gender, Writing, and Le Roman de Silence », 1989, p. 109.

6. Voir notre bibliographie, où l'on trouvera des tenants de toutes les positions interprétatives, qui penchant plutôt pour la misogynie (M. Perret, S. Gaunt), qui pour le féminisme (notamment K. J. Brahney, A. Benaim Lasry et R. Psaki, dans l'introduction à sa traduction), qui pour la réaction féministe induite par la misogynie (c'est la solution, assez astucieuse, de R. L. Krueger), qui pour l'incertitude et l'ambiguïté idéologiques (P. L. Allen, R. Bloch) etc. 
7. Sur le rôle que peut jouer le public féminin de l'auteur dans le sens à donner au roman, voir l'intéressante interprétation, déjà évoquée plus haut, de R. L. Krueger, dans Women Readers and the Ideology of Gender in Old French Verse Romance, 1993, p. 101-127.

8. Sur ces textes, envisagés dans leurs rapports au Roman de Silence, voir M. Perret, « Travesties et transsexuelles : Yde, Silence, Grisandole, Blanchandine », 1985.

9. Voir Tristan de Nanteuil, éd. K. V. Sinclair, Assen, Van Gorcum, 1971 et Esclarmonde, Clarisse et Florent, Yde et Olive, éd. M. Schweigel, Marburg, 1889. Précisons que ce n'est pas par hasard que j'ai aussi mentionné plus haut Aucassin et Nicolette, où l'épisode d'Yde et Olive trouve peut-être son origine, étant donné le rôle évident joué par la cantefable dans l'écriture d'une bonne partie de Clarisse et Florent.

10. On trouve l'histoire de Grisandole dans The Vulgate Version of the Arthurian Romance, tome deux, L'Estoire de Merlin, éd. H. O. Sommer, Washington, 1908, p.281-292 ou dans la partie intitulée Les Premiers faits du roi Arthur du Livre du Graal, éd. et trad. P. Walter et alii, Paris, Gallimard, 2001, tome un, p. 1226-1253.

11. Plus largement, sur les liens entre cet épisode et le reste du Lancelot-Graal, voir R. Trachsler, «Merlin chez Jules César. De l'épisode de Grisandole à la tradition manuscrite de la suite du Merlin ", Studi francesi, 133, 2001, p. 61-71.

12. Voir Le Jongleur dans la littérature narrative des XII et XIII ${ }^{e}$ siècles (Paris, Champion, 2005) le chapitre VI intitulé «Évaluations ». Les remarques qui y sont développées concernant l'idéologie dans le texte s'appuient essentiellement sur les ouvrages de P. Hamon, Texte et idéologie (Paris, PUF, $1^{\text {re }}$ éd. 1984), de V. Jouve, Poétique des valeurs (Paris, PUF, 2001), et sur celui déjà cité de S.R. Suleiman.

13. Sur ce point, voir plus particulièrement W. C. Booth, The Rhetoric of Fiction, University of Chicago, $1^{\text {re éd. }} 1961$.

14. Voir sur ce point les remarques de V. Jouve, Poétique des valeurs, p. 118-124.

15. G. Genette, « Discours du récit », Figures III, Paris, Seuil, 1972, p. 263.

16. V. Jouve, Poétique des valeurs, p. 93.

17. Le rôle de Merlin dans Le Roman de Silence a récemment été étudié par P. Clements («ShapeShifting and Gender-Bending: Merlin's Last Laugh at Silence », 1998), S. Roche-Mahdi («A Reappraisal of the Role of Merlin in the Roman de Silence ») et L. Koschanske Stock («Civilization and Its Discontents : Cultural Primitivism and Merlin as a Wild Man in the Roman de Silence »), ces deux derniers articles (dont on recommandera particulièrement la lecture) dans Arthuriana. Special Issue : Essays on Le Roman de Silence, 2002.

18. C'est aussi le rôle de Merlin dans un des rares autres romans en vers à faire une place à ce personnage, Claris et Laris. Voir sur ce point P.Zumthor, Merlin le prophète, Lausanne, 1943 (réimp. Slatkine, 1973), p. 258 (note 1). De fait, dans Claris et Laris, l'unique apparition de Merlin n'a d'autre rôle que de relancer une action qui resterait bloquée sans son intervention magique. C'est l'exemple caractéristique d'un roman où, selon les propres termes de P. Zumthor, Merlin « apparaît stupidement comme un deux ex machina pour faire progresser l'action » (op. cit., p. 273). 19. Pour une comparaison détaillée entre ces deux textes, voir notamment L. Thorpe, dans l'introduction à son édition (p. 27-32) et dans « Merlin's Sardonic Laughter » (Studies in medieval literature and languages in memory of Frederick Whitehead, éd. W. Rothwell et alii, Manchester University, 1973, tome I, p. 323-339), ainsi que D. James-Raoul, « Un curieux avatar de L'Estoire Merlin : Le Roman de Silence », 1996.

20. La critique apparaît assez divisée sur ce point : à l'avis très tranché de L. Thorpe, et dans une moindre mesure de D. James-Raoul, en faveur de la dépendance directe du Roman de Silence à l'histoire de Grisandole, on peut opposer celui de F. Lecoy, selon lequel les deux textes pourraient dériver d'une source commune (voir "Corrections : Le Roman de Silence d'Heldris de Cornualle », 1978, p. 110), ou celui de H. Gelzer («Der Silenceroman von Heldris de Cornualle», 1927), qui reste très prudent sur ce point. 
21. Voir la récente édition, qui comporte texte latin et traduction, réalisée sous la direction de Ph. Walter, Le Devin maudit. Merlin, Lailoken, Suibhne, Grenoble, ELLUG, 1999.

22. Notons qu'en ce point précis du texte Heldris s'écarte assez notablement de la tradition merlinienne. Rappelant par la bouche d'Eufeme l'histoire de la tour de Vortigern (v. 5784-5803), Heldris lui donne une conclusion inhabituelle. Dès la construction de la tour achevée, alors qu'il n'est encore que « petis enfes» (v. 5794), Merlin s'en va vivre dans les bois, particularité absente des autres textes relatant la même anecdote (voir aussi les vers 5908-5911). C'est peut-être la façon pour Heldris d'abord de montrer qu'il n'est en rien esclave de la tradition, et aussi d'attirer l'attention sur cet état d'homme sauvage si caractéristique du personnage de Merlin qu'il ne l'a pas quitté depuis son plus jeune âge.

23. Les seules allusions à la folie que comporte le texte apparaissent dans deux comparaisons : pour s'en nourrir, Merlin saisit la viande cuite "com sil fust forsenes"; puis, enchaîné au moment de sa capture, il se débat « comme derves » (éd. H. O. Sommer, p. 284). Mais une fois à la cour de César, Merlin n'est ni emprisonné, ni menacé de mort.

24. P. Walter, Merlin ou le savoir du monde, Paris, Imago, 2000, p. 76. Les multiples aspects contradictoires $d u$ personnage, son caractère protéen etc. sont particulièrement bien décrits dans cet ouvrage, notamment dans les chapitres consacré à «Merlin, l'enfant-vieillard " et à «Merlin Protée». Sur Merlin, on peut aussi se reporter à P.Zumthor, Merlin le prophète, et à F. Dubost, Aspects fantastiques de la littérature narrative médiévale (XII ${ }^{e}$-XIII ${ }^{e}$ siècles), Paris, Champion, 1991, p. 711-751.

25. Ce détail significatif a été relevé par S. Conklin Akbari, «Nature's Forge Recast in the Roman de Silence ", 1994, p. 44.

26. Nous empruntons cette référence à La Poétique du merveilleux dans le Lancelot en prose (Paris, Champion, 1998) de J.-R. Valette et renvoyons aux pages 109-143 de son ouvrage pour un exposé plus détaillé des théories de L. Lévy-Bruhl, plus précisément appliquées au merveilleux, mais qui n'en sont pas moins éclairantes quant à la mentalité médiévale en général.

27. M. Éliade, "Méphistophélès et l'androgyne», dans Méphistophélès et l'androgyne, Paris, Gallimard, 1962, p. 111-179.

28. La notion de coincidentia oppositorum, telle qu'elle est envisagée dans "Méphistophélès et l'androgyne ", peut ouvrir à des perspectives intéressantes pour la compréhension du Roman de Silence au niveau de la "pensée sauvage» ou mythique (sans qu'il s'agisse, cependant, de cantonner la notion de coincidentia oppositorum à ce niveau, dans la mesure où elle entre aussi, comme le rappelle M. Éliade, dans la pensée philosophique d'un Jean Scot Érigène ou d'un Nicolas de Cuse), perspectives que nous ne pouvons développer ici. En ce qui concerne Merlin, on se contentera de renvoyer à l'ouvrage déjà cité de P. Walter, où le personnage est particulièrement abordé sous cet angle, en soulignant seulement l'intérêt spécial que présente Le Roman de Silence quant au rire carnavalesque de Merlin (voir P. Walter, op. cit., p. 147-157), dont la mise en scène par Heldris relève d'un véritable complexe d'oppositions (folie opposée à la sagesse, silence opposé à la parole, rire opposé à la triste réalité etc.). Quant à Silence, le personnage mériterait d'être envisagé plus précisément, à partir du cadre posé par M. Éliade, dans la perspective d'une androgynie à caractère rituel et initiatique, à la fois comme aboutissement, Silence étant à l'image de l'être parfait totalisant le masculin et le féminin, et comme étape transitoire d'indifférenciation ouvrant à une différenciation pleinement assumée, Silence devenant ainsi l'exemple d'une femme parfaite dans la mesure où elle a aussi été homme (voir M. Éliade, qui évoque divers cas où « l'initation de puberté implique l'androgynisation préalable du néophyte ", car « on ne peut devenir un être sexuellement adulte avant d'avoir connu la coexistence des sexes, l'androgynie ; autrement dit, on ne peut pas accéder à un mode d'être particulier et bien déterminé avant d'avoir connu un mode d'être total », p. 161). Sur l'androgynie dans Le Roman de Silence, et ses liens possibles avec la pensée de Jean Scot Érigène, voir P. Victorin, « Le nu et le 
vêtu dans Le Roman de Silence: métaphore de l'opposition entre nature et norreture ", 2001, en particulier p. 377-379.

29. Sur les relations entre Merlin et l'université, notamment avec sa prophétie sur la future floraison des sciences à Oxford, voir M. Stanesco, « Nigromance et Université. Scolastique du merveilleux dans le roman français du Moyen Âge», p. 261-262. Rappelons aussi que la Vita Merlini comporte plusieurs longs développements à caractère encyclopédique.

30. B.C. Bazàn, "Les questions disputées, principalement dans les facultés de théologie ", Les Questions disputées et les questions quodlibétiques dans les facultés de théologie, de droit et de médecine, Turnhout, Brepols, Typologie des sources du Moyen Âge occidental n 44-45, 1985, p. 146. Nous tirons toutes nos remarques sur la quaestio de cet ouvrage.

31. «La question, comme le note F. Bouchet, à la mode au XIII siècle, est controversée. Témoins, de nombreux proverbes qui énoncent tantôt la primauté de Nature, tantôt celle de Culture » (voir l'introduction à sa traduction dans Récits d'amour et de chevalerie, 2000, p. 462).

32. H. Lloyd, "The Triumph of Pragmatism : Reward and Punishment in Le Roman de Silence ", 1987.

33. Pragmatisme, autrement dit, pour reprendre une partie de la définition proposée par le Vocabulaire technique et critique de la philosophie de Lalande, "doctrine selon laquelle la vérité est une relation entièrement immanente à l'expérience humaine [...]; la vérité d'une proposition consiste donc dans le fait qu'elle "est utile", qu'elle "réussit", qu'elle "donne satisfaction" ".

34. Guillaume de Lorris et Jean de Meun, Le Roman de la Rose, éd. et trad. A. Strubel, Paris, Le Livre de Poche, 1992.

35. Voir K. Pratt, « Humour in the Roman de Silence », 2003.

\section{AUTEUR}

\section{SILVÈRE MENEGALDO}

